\title{
Variabilidade Metodológica na Aquisição, Reconstrução e Análise de Estudos de PET/CT Oncológico com FDG- ${ }^{18} \mathrm{~F}$ : Levantamento Estadual Variability in ${ }^{18} \mathrm{~F}$-FDG PET/CT Methodology of Acquisition, Reconstruction and Analysis for Oncologic Imaging: State Survey
}

\author{
Andréia C. F. da S. Fischer ${ }^{1,4}$, Aline C. Druziann, ${ }^{1,2}$ Diego B. Pianta ${ }^{3}$ \\ Ana M. Marques da Silva ${ }^{4}$, Alexandre Bacelar ${ }^{1}$ \\ ${ }^{1}$ Serviço de Física Médica e Radioproteção, Hospital de Clínicas de Porto Alegre, Porto Alegre, Brasil \\ ${ }^{2}$ Faculdade de Física Médica, UFCSPA, Porto Alegre, Brasil \\ ${ }^{3}$ Serviço de Medicina Nuclear e PET/CT, Hospital de Clínicas de Porto Alegre, Porto Alegre, Brasil \\ ${ }^{4}$ Núcleo de Pesquisas em Imagens Médicas (NIMed)/Faculdade de Física, PUCRS, Porto Alegre, Brasil
}

\begin{abstract}
Resumo
A quantificação do SUV em PET/CT oncológico com FDG- ${ }^{18} \mathrm{~F}$ mostra-se útil para o diagnóstico, estadiamento e avaliação de tratamentos em oncologia. Entretanto, fatores biológicos e técnicos acarretam imprecisão à sua quantificação. O uso do SUV como ferramenta diagnóstica exige que a influência destes seja minimizada mediante a padronização dos métodos de aquisição, reconstrução e análise dos estudos. Este trabalho tem por objetivo avaliar a variabilidade metodológica da aquisição de estudos de PET/CT no Estado do Rio Grande do Sul. Para tanto, foi aplicado um questionário para levantamento das informações técnicas dos sistemas PET/CT e dos métodos de aquisição e análise de exames. Todos os serviços implementam programas de garantia da qualidade coerentes com as recomendações (inter)nacionais. Entretanto, os métodos de aquisição e reconstrução da PET divergem. Sugere-se a implementação de uma estratégia de harmonização da quantificação do SUV, atribuindo-Ihe maior reprodutibilidade e repetibilidade.
\end{abstract} Palavras-chave: PET/CT; protocolos de imagem; padronização; SUV.

\section{Abstract}

The SUV in ${ }^{18} \mathrm{~F}-F D G$ PET/CT oncological imaging is useful for cancer diagnosis, staging and treatment assessment. There are, however, several factors that can give rise to bias in SUV measurements. When using SUV as a diagnostic tool, one needs to minimize the variability in this measurement by standardization of patient preparation, acquisition and reconstruction parameters. The aim of this study is to evaluate the methodological variability in PET/CT acquisition in Rio Grande do Sul State. For that, in each department, a questionnaire was applied to survey technical information from PET/CT systems and about the acquisitions and analysis methods utilized. All departments implement quality assurance programs consistent with (inter)national recommendations. However, the acquisition and reconstruction methods of acquired PET data differ. The implementation of a harmonized strategy for quantifying the SUV is suggested, in order to obtain greater reproducibility and repeatability.

Keywords: PET/CT; imaging protocols; standardization, SUV.

\section{Introdução}

A tomografia por emissão de pósitrons ou PET (Positron Emission Tomography) é uma modalidade de imagem molecular utilizada para avaliação in vivo da atividade metabólica. Esta técnica utiliza radiofármacos emissores de pósitrons, tais como o FDG- ${ }^{18} \mathrm{~F}$ (Fluordesoxiglicose), que permitem a localização de tecidos com alta atividade metabólica, incluindo tumores ${ }^{1}$.

Em sistemas híbridos de PET/CT (Positron Emission Tomography/Computed Tomography) as informações metabólicas da imagem de PET são adquiridas conjuntamente com imagens anatômicas, através de uma aquisição de tomografia computadorizada por raios $X$ de baixa dose para a realização da correção de atenuação das imagens PET.

$\mathrm{Na}$ rotina clínica, as imagens de PET/CT costumam ser analisadas qualitativamente, mediante a comparação visual entre a captação nas lesões tumorais e nos tecidos normais; e, semiquantitativamente, por meio de um parâmetro denominado SUV (Standardized Uptake Values, Valores Padronizados de Captação, em $\mathrm{g} / \mathrm{mL}$ ), definido como a razão entre a concentração de atividade no tecido $(\mathrm{kBg} / \mathrm{mL})$ e a atividade injetada por massa do paciente $(\mathrm{kBq} / \mathrm{g})$, corrigida para 0 decaimento radioativo ${ }^{1}$.

A quantificação de lesões tumorais em imagens de PET/CT com FDG- ${ }^{18} \mathrm{~F}$ utilizando o SUV mostrase útil tanto para o diagnóstico, quanto para 0 estadiamento, avaliação da resposta e acompanhamento de tratamentos em oncologia ${ }^{1,2,3}$. Porém, pesquisas relatam que os fatores que afetam tal quantificação são diversos $2,4,5,6,7$. Dentre os aspectos mais relevantes, destacam-se: (1) fatores biológicos, diretamente ligados ao preparo do paciente e execução do exame, tais como a 
preparação e metabolismo individual do paciente, o tempo de captação e a movimentação involuntária durante a aquisição dos estudos; e (2) fatores técnicos, nos quais se enquadram os protocolos de aquisição, métodos e parâmetros de reconstrução empregados, metodologia de análise e a calibração do equipamento ${ }^{8}$ inclusive. $\mathrm{Em}$ estudos multicêntricos ainda há o agravante de diversos equipamentos serem utilizados. Sensibilidade, correções aplicadas aos dados brutos e/ou algoritmos e parâmetros de reconstrução podem divergir substancialmente entre sistemas de $\mathrm{PET} / \mathrm{CT}$, o que pode afetar, diretamente, a acurácia e precisão da quantificação do SUV em até $23,0 \% 5,6$.

Assim, para que o SUV possa ser utilizado como ferramenta diagnóstica e prognóstica, faz-se necessária e imprescindível a minimização da variabilidade atribuída à sua quantificação $0^{9,10,11}$. Isso pode ser alcançado mediante a padronização das etapas de estudos de PET/CT com FDG-18 ${ }^{11}$, de maneira que as informações quantitativas obtidas nesses estudos sejam reprodutíveis e repetíveis. A reprodutibilidade refere-se à habilidade de atingir os mesmos resultados quantitativos em um único paciente quando esse é examinado em diferentes sistemas/instituições; enquanto a repetibilidade diz respeito a atingir os mesmos resultados em diferentes estudos, de um determinado paciente, adquiridos em um só equipamento ${ }^{12}$.

Procedimentos padronizados promovem maior consistência às informações quantitativas obtidas em exames de PET/CT em diferentes estudos, plataformas de análise e instituições. Nesse sentido, diversas organizações e sociedades desenvolveram guias ou recomendações (guidelines) em relação à padronização dos exames de PET/CT $3,12,13,14,15,16,17,18,19$, englobando, dentre outros aspectos, os procedimentos recomendados para aquisição e reconstrução dos dados e métodos de visualização e análise das imagens.

Este estudo tem por objetivo avaliar a variabilidade metodológica da aquisição de estudos de PET/CT no Estado do Rio Grande do Sul (RS), focando-se nos fatores técnicos. Quando possível, os procedimentos aplicados serão comparados com aqueles recomendados pelos guidelines $3,12,13,14$.

\section{Materiais e Métodos}

Para realizar a avaliação, foram apuradas as características técnicas dos equipamentos e protocolos de aquisição e análise das imagens de distintos serviços de PET/CT (doravante denominados SMN) instalados no RS, mediante a aplicação de um questionário para o levantamento de dados. $O$ questionário, desenvolvido anteriormente por Fischer $(2014)^{20}$, foi enviado ao responsável técnico ou físico de cada instalação que possuísse equipamentos de PET/CT em operação no RS, por e-mail e/ou de forma impressa.

Esse instrumento é composto por questões de múltipla escolha, questões abertas e/ou lacunas a serem preenchidas. São solicitadas informações técnicas sobre o sistema PET/CT e o medidor de atividade, tais como marca, modelo, ano de instalação e garantia da qualidade; parâmetros de aquisição e reconstrução dos dados utilizados na prática clínica; programas e métodos utilizados para visualização e análise das imagens obtidas; e as características do SMN.

Para a análise dos dados coletados, as questões foram agrupadas em categorias, conforme sumário exposto na Figura 1, de maneira a simplificar a comparação dos dados coletados com os guidelines da Associação Europeia de Medicina Nuclear $^{12}$ (EANM, European Association of Nuclear Medicine), do Colégio Americano de Radiologia ${ }^{13}$ (ACR, American College of Radiology), da Sociedade Brasileira de Medicina Nuclear ${ }^{14}$ (SBMN) e da Sociedade de Medicina Nuclear e Imagem Molecular ${ }^{3}$ americana (SNMMI, Society of Nuclear Medicine and Molecular Imaging).

\section{Resultados e Discussões}

O estado do RS possuía até abril de 2016 - data de submissão desse artigo - oito instalações com equipamentos de PET/CT. Contudo, o estudo abrange seis dessas instalações (75\%). Apesar de não ter sido possível contemplar todos os SMN do RS a amostra contempla, no mínimo, um SMN de cada região do estado em que há este tipo de equipamento instalado. Os resultados estão descritos nas subseções a seguir.

\subsection{Demografia}

Os responsáveis pelo preenchimento do questionário foram: físicos $(83,3 \%)$ e médicos nucleares $(16,7 \%)$.

Os SMN localizam-se, em sua maioria (quatro), em Porto Alegre. As outras duas instalações encontram-se na região metropolitana de Porto Alegre e na Serra Gaúcha.

A metade dos SMN do RS possui natureza de estabelecimento de "pessoa jurídica instituída exclusivamente para fins filantrópicos", ou seja, são entidades beneficentes sem fins lucrativos. Apenas um SMN possui natureza de "hospital público integrante do Sistema Único de Saúde (SUS)" e os outros dois classificam-se como "outros". Apenas um dos seis estabelecimentos não possui habilitação para realização de exames de PET/CT no âmbito do SUS 21 .

No que tange ao regime jurídico, somente uma instalação apresenta regime "público". O restante enquadra-se em regime "privado". 


\begin{tabular}{ll}
\hline \multicolumn{1}{c}{ Categoria } & \multicolumn{1}{c}{ Questões } \\
\hline \hline Demografia & $\begin{array}{l}\text { Qualificação do responsável pelo preenchimento } \\
\text { Localização do SMN } \\
\text { Natureza e regime jurídico do estabelecimento }\end{array}$ \\
\hline Informações Técnicas & $\begin{array}{l}\text { Fabricante e modelo do PET/CT } \\
\text { Data de instalação do equipamento } \\
\end{array}$ \\
& Manutenção Preventiva: Que frequência é realizada? Qual a data da última manutenção? \\
& Medidor de Atividade: Específico para PET? Quando foi instalado? \\
Garantia da Qualidade & Frequência da realização dos testes do PET/CT e do Medidor de Atividade \\
& Já foram realizados os testes do NEMA NU 2-2007? \\
Parâmetros de aquisição/ & Frequência da sincronização dos horários entre o medidor de atividade e o PET/CT \\
reconstrução dos dados & Parâmetros de aquisição: Tempo/posição da maca, FOV, matriz, Atividade Administrada. \\
Visualização e Análise das & Algoritmo e Parâmetros de Reconstrução: Implementado PSF modelling? ToF disponível? \\
Imagens & Softwares utilizados \\
& Método de delimitação da ROI/VOI para o cálculo do SUV: 2D ou 3D? \\
& Método de determinação do SUV: SUX
\end{tabular}

Figura 1. Sumário das questões realizadas no levantamento de dados.

\subsection{Informações Técnicas}

A amostra abrange todos os fabricantes que atualmente comercializam sistemas de PET/CT no Brasil (Philips Medical Systems, General Eletric Medical System e Siemens Medical Solutions). O número total de equipamentos é igualmente distribuído por fabricante (33,3\% cada).

A ordem cronológica de início de operação de cada SMN encontra-se na Figura 2. Nenhum equipamento sofreu upgrade, nem de software nem mecânico, desde as suas instalações.

\begin{tabular}{ccccccc}
\hline SMN & RS- & RS- & RS- & RS- & RS- & RS- \\
& $\mathbf{1}$ & $\mathbf{2}$ & $\mathbf{3}$ & $\mathbf{4}$ & $\mathbf{5}$ & $\mathbf{6}$ \\
\hline \hline Ano de & 200 & 201 & 201 & 201 & 201 & 201 \\
instalação & 8 & 0 & 1 & 2 & 4 & 5 \\
\hline
\end{tabular}

Figura 2. Quadro da ordem cronológica de início de operação dos equipamentos de PET/CT, onde RS-n refere-se a cada (n) SMN.

Pode-se observar que o mercado estadual do RS de equipamentos de PET/CT encontra-se em franca expansão. O primeiro sistema foi instalado em 2008, na capital Porto Alegre. No período de 2010 a março de 2015, entraram em funcionamento outras sete instalações com esta tecnologia, das quais cinco participaram deste levantamento.

Portanto, já existem pacientes que transitam entre as diferentes instituições do estado para a realização de seus estudos longitudinais. Nesses casos, a mudança percentual/relativa do SUV em estudos, realizados não necessariamente no mesmo equipamento, é analisada para avaliação da resposta do indivíduo ao tratamento ${ }^{10}$. Deste modo, corrobora-se a necessidade de que os exames de $\mathrm{PET} / \mathrm{CT}$ sejam adquiridos de uma maneira consistente e padronizada, minimizando a variabilidade metodológica dentre as instituições envolvidas e, consequentemente, atribuindo maior reprodutibilidade à quantificação do SUV.

Em relação ao programa de manutenção preventiva do equipamento de PET/CT, todos os SMN investigados possuem um programa ativo, diferindo unicamente em sua periodicidade: $66,7 \%$ possuem manutenções semestrais; os demais $(33,3 \%)$, frequência anual.
Ao que se refere ao medidor de atividade, todos os SMN possuem equipamentos adequados do ponto de vista técnico, ou seja, possuem medidores compatíveis com a energia dos radioisótopos utilizados em $\mathrm{PET}^{22}$.

\subsection{Garantia da Qualidade}

A seguir estão descritos os resultados acerca do controle da qualidade do equipamento de PET/CT e do medidor de atividade dos SMN.

\subsubsection{PET/CT:}

Todos os SMN, com apenas uma exceção, realizam semestralmente os testes descritos no protocolo "NEMA NU 2-2007: Performance Measurements of Positron Emission Tomographs"23. Esse protocolo propõe uma metodologia padrão de mensuração das características básicas de desempenho de um equipamento de PET, tais como sensibilidade, resolução espacial, NECR (Noise Equivalent Count Rate, taxa de contagem de ruído equivalente), fração de espalhamento e qualidade da imagem. Ele é uma ferramenta adequada para averiguar se o equipamento está operando de acordo com as especificações do fabricante ${ }^{9}$, um pré-requisito técnico para a realização de exames de PET/CT quantitativos ${ }^{11}$.

Calibração do SUV. Dos SMN investigados, metade realiza a Calibração do SUV semestralmente e/ou após reparo e, a outra metade, trimestralmente. Todos os SMN efetuam a Verificação da Calibração trimestralmente.

A calibração do SUV (também chamada de Cross Calibration ou Calibração da Concentração Radioativa ${ }^{22}$ ) é outro pré-requisito técnico para aquisição de imagens PET quantitativas ${ }^{9}$. Nesse procedimento, onde se realiza a aquisição de um simulador cilíndrico uniforme preenchido com água contendo uma quantidade de material radioativo conhecida e mensurada pelo medidor de atividade da instituição, as unidades arbitrárias do equipamento são convertidas para concentração radioativa, possibilitando a quantificação da 
concentração de radioatividade nas imagens de $\mathrm{PET}^{7}$. Uma calibração imprópria pode acarretar erros na quantificação do SUV com magnitude de $10 \% 9$

Esta calibração, em princípio, corrige as variações globais de sensibilidade do equipamento, garantindo a acurácia do SUV. De acordo com a legislação brasileira ${ }^{22}$ e o guideline europeu ${ }^{12}$ esse procedimento deve ser realizado trimestralmente. Entretanto, grande parte dos fabricantes recomenda que a periodicidade seja semestral e/ou após reparos. Logo, a calibração não compensaria possíveis variações ocorridas durante o intervalo entre as calibrações ${ }^{24}$.

Para garantir a acurácia do SUV neste intervalo de tempo, realiza-se o teste de Verificação da Calibração do sistema, cuja frequência recomendada pelo fabricante é menor, usualmente trimestral (frequência esta praticada por todos os SMN investigados). Caso sejam encontradas discrepâncias no valor de SUV (SUV mensurado na água diferente de $1 \pm 10 \%)$, torna-se necessária nova Calibração.

Assim, apesar de não atenderem ao exposto na legislação brasileira ${ }^{22}$, os $S M N$, que realizam semestralmente a Calibração do SUV, verificam a exatidão da quantificação mediante a realização trimestral do teste de Verificação. Contudo, no questionário aplicado neste estudo ${ }^{20}$ não se questionou os valores obtidos nos testes de Verificação/Calibração do SUV, apenas a periodicidade de realização desses. Desta maneira, não pode-se assegurar a acurácia do SUV dentre os SMN investigados.

Varredura em branco. Todos os SMN realizam a aquisição da varredura em branco (também chamada de blank scan ${ }^{22}$ ) diariamente, antes de administrar o material radioativo aos pacientes. Esse procedimento garante o correto funcionamento dos módulos detectores, identificando falhas nos mesmos e/ou flutuações nos componentes eletrônicos ${ }^{12}$, sendo recomendado pela totalidade dos guidelines internacionais revisados neste trabalho ${ }^{3,12,13}$.

\subsubsection{Medidor de Atividade:}

Os testes de controle de qualidade do medidor de atividade são realizados, por todos os SMN, em concordância com 0 exigido pela legislação nacional 22,25 .

Todas as instituições investigadas verificam a sincronização dos relógios do equipamento de PET/CT e do medidor de atividade. Dois SMN realizam a verificação diariamente; dois semanalmente; e os outros dois, mensalmente.

Esta prática não é exigida na legislação brasileira 22,25 . Dos guidelines revisados $3,12,13,14$, apenas o europeu ${ }^{12}$ recomenda que a verificação da sincronização dos horários seja realizada regularmente, sendo admitida uma discrepância máxima de um (01) minuto. Sugere-se que essa verificação seja incluída na legislação brasileira, pois uma discrepância de oito (08) minutos entre os relógios dos equipamentos pode acarretar erros no SUV acima de $5 \%$ devido à correção incorreta do decaimento radioativo $0^{5,7,9}$.

\subsection{Parâmetros de aquisição/reconstrução}

$A$ atividade de FDG- ${ }^{18} \mathrm{~F}$ administrada baseia-se na massa do paciente, adulto ou pediátrico, em $100 \%$ dos SMN. A Figura 3 apresenta um quadro comparativo das recomendações dos Guidelines 3,12,13,14 com os resultados obtidos.

Para adultos, a atividade média injetada foi igual a $4,7 \mathrm{MBq} / \mathrm{kg}(0,127 \mathrm{mCi} / \mathrm{kg})$, variando entre 4,07$5,55 \mathrm{MBq} / \mathrm{kg}(0,11-0,15 \mathrm{mCi} / \mathrm{kg})$, de acordo com os guidelines europeu ${ }^{12}$ e brasileiro ${ }^{14}$ que consideram, dentre outros fatores, a massa do paciente para o cálculo da atividade. Os guias da SNMMI ${ }^{3}$ e $\mathrm{ACR}^{11}$ recomendam que seja administrada uma atividade padrão, entre $370-740 \mathrm{MBq}(10-20 \mathrm{mCi})$, a todos os pacientes adultos, o que vai de encontro ao princípio de otimização de proteção radiológica.

Em crianças, a média de atividade administrada obtida foi ligeiramente maior: 5,7 MBq/kg $(0,153 \mathrm{mCi} / \mathrm{kg})$, variando entre 4,07-7,40 MBq/kg $(0,11-0,2 \mathrm{mCi} / \mathrm{kg})$. Tais valores estão em conformidade com os guias revisados $3,12,13,14$.

\begin{tabular}{|c|c|c|c|c|c|}
\hline Parâmetro & $\mathrm{ACR}^{13}$ & SNMMI $^{3}$ & EANM $^{12}$ & SBMN $^{14}$ & $\begin{array}{c}\text { Resultados } \\
\text { Obtidos }\end{array}$ \\
\hline \multirow[t]{2}{*}{$\begin{array}{l}\text { Atividade } \\
\text { administrada }\end{array}$} & $\begin{array}{l}370-740 \mathrm{MBq} \\
(10-20 \mathrm{mCi})\end{array}$ & $\begin{array}{c}370-740 \mathrm{MBq} \\
(10-20 \mathrm{mCi})\end{array}$ & $\begin{array}{c}\text { De acordo com o eq. }{ }^{[1]}, \\
\text { tempo/posição da maca } \\
\text { e peso do paciente; } \\
A_{\text {máx }}=14 \mathrm{mCi}\end{array}$ & $\begin{array}{c}370 \mathrm{MBq}(10 \mathrm{mCi}) \\
\text { ou } 4,44-5,18 \mathrm{MBq} / \mathrm{kg} \\
(0,12-0,14 \mathrm{mCi} / \mathrm{kg})\end{array}$ & $\begin{array}{r}4,07-5,55 \mathrm{MBq} / \mathrm{kg} \\
(0,11-0,15 \mathrm{mCi} / \mathrm{kg})\end{array}$ \\
\hline & $\begin{array}{l}3,7-5,2 \mathrm{MBq} / \mathrm{kg} \\
(0,10-0,14 \mathrm{mCi})\end{array}$ & $\begin{array}{c}5,18-7,4 \mathrm{MBq} / \mathrm{kg} \\
(0,14-0,20 \mathrm{mCi} / \mathrm{kg})\end{array}$ & $\begin{array}{l}\text { De acordo com EANM } \\
\qquad(2006)^{26}\end{array}$ & $\begin{array}{c}4,44-5,18 \mathrm{kBq} / \mathrm{kg} \\
(0,12-0,14 \mathrm{mCi} / \mathrm{kg})\end{array}$ & $\begin{array}{l}4,07-7,40 \mathrm{MBq} / \mathrm{kg} \\
(0,11-0,2 \mathrm{mCi} / \mathrm{kg})\end{array}$ \\
\hline $\begin{array}{l}\text { Tempo/posição da } \\
\text { maca }\end{array}$ & $1-5 \min$ & $2-5 \min$ & $\begin{array}{c}\text { De acordo com a } \\
\text { Atividade Administrada }\end{array}$ & $2-5 \min$ & $1: 45-3 \mathrm{~min}$ \\
\hline $\begin{array}{l}\text { Reconstrução dos } \\
\text { dados PET }\end{array}$ & $\begin{array}{c}\text { Não } \\
\text { mencionado }\end{array}$ & $\begin{array}{l}\text { Preferência aos } \\
\text { métodos } \\
\text { iterativos }\end{array}$ & $\begin{array}{c}\text { Parâmetros devem ser } \\
\text { escolhidos de maneira } \\
\text { que a imagem final } \\
\text { atinja as } \\
\text { especificações de } \\
\text { harmonização } \\
\text { propostas pela EARL }{ }^{27}\end{array}$ & Não mencionado & $\begin{array}{c}\text { Métodos } \\
\text { iterativos } \\
\text { (recomendados } \\
\text { pelo fabricante) }\end{array}$ \\
\hline
\end{tabular}

[1] De acordo com a sobreposição entre as posições da maca.

Figura 3. Quadro comparativo dos resultados obtidos com as recomendações dos guidelines revisados ${ }^{3,12,13,14}$. 

Os parâmetros de aquisição e reconstrução de estudos de PET/CT oncológico, empregado em cada SMN participante deste estudo, encontram-se na

Figura 4. Pode-se perceber a preocupação dos SMN quanto à otimização dos protocolos de aquisição da tomografia computadorizada. $66,7 \%$ das instituições investigadas empregam ferramentas que modificam automaticamente a corrente da exposição, de acordo com o tamanho e formato do paciente, visando à redução da dose absorvida pelo paciente.

Dos fatores técnicos que atribuem maior variabilidade à quantificação do SUV, os mais proeminentes são os parâmetros de aquisição e reconstrução dos dados da PET em decorrência das suas implicações na resolução espacial da imagem obtida ${ }^{9,10}$. Mudanças de parâmetros de reconstrução como tamanho da matriz, FOV (Field Of View) e o emprego das tecnologias ToF (Timeof-Flight) e/ou PSF (Point-Spread Function) podem alterar significativamente os valores de SUV4,5,6,7. Dentre os SMN investigados, observa-se que são empregados diferentes tamanhos de FOV e métodos de reconstrução distintos. Há instituições que dispõem da tecnologia PSF (33,3\%) e até mesmo ToF (16,67\%). Em um âmbito multicêntrico, é necessária a padronização destes parâmetros a fim de reduzir tal variabilidade. Entretanto, a definição de um protocolo de aquisição e reconstrução das imagens único a todos os equipamentos de PET/CT é inviável, uma vez que cada fabricante implementa algoritmos de reconstrução proprietários ou de maneira diferente ${ }^{11}$.

Assim, surge o conceito de harmonização, onde os parâmetros de desempenho ou características da imagem são definidos a posteriori, a fim de determinar as configurações de aquisição, processamento e análise necessárias para que os diferentes sistemas de PET/CT gerem, individualmente, imagens cujas características convirjam para aquelas geradas pela maior parte dos equipamentos ${ }^{9,11}$.

Esta abordagem visa minimizar as diferenças da qualidade da imagem e a variabilidade da quantificação do SUV entre sujeitos, equipamentos e instituições, mediante a aquisição de estudos com características da imagem equiparáveis ${ }^{11}$.

Uma estratégia de harmonização da quantificação do SUV em imagens de PET/CT, baseada no programa de acreditação "EARL FDGPET/CT Accreditation Programme"27 do Grupo de Imagens da Organização Europeia para Pesquisa e Tratamento do Câncer (European Organisation for Research and Treatment of Cancer - EORTC Imaging Group), é proposta por Fischer20. A estratégia, realizada com equipamentos de diferentes modelos e fabricantes, tem como objetivo identificar os parâmetros de reconstrução adequados à harmonização da quantificação, em nível regional e internacional, em cada equipamento analisado. Como resultado da estratégia, identificam-se as especificações de desempenho harmonizáveis dentre os equipamentos avaliados e os parâmetros de reconstrução mais adequados à harmonização da quantificação.

\subsection{Visualização e Análise das Imagens}

Os SMN investigados utilizam, em sua totalidade, a estação de trabalho (workstation) do fabricante do equipamento de PET/CT para visualização e análise das imagens adquiridas. Metade utiliza, adicionalmente, o programa OsiriX. Plataformas PACS (sistema de comunicação e arquivamento de imagens, Picture Archiving and Communication System) são utilizadas por dois SMN exclusivamente para visualização das imagens.

\begin{tabular}{|c|c|c|c|c|c|c|}
\hline & \multicolumn{6}{|c|}{ INSTALAÇÕES } \\
\hline Parâmetro & RS-1 & RS-2 & RS-3 & RS-4 & RS-5 & RS-6 \\
\hline \multicolumn{7}{|c|}{ PET $^{[1]}$} \\
\hline $\begin{array}{l}\text { Tempo/posição } \\
\text { da maca (min) }\end{array}$ & $01: 45$ & 03:00 & 03:00 & 02:30 & 02:00 & 02:00 \\
\hline Matriz & 144 & 144 & 168 & 192 & 168 & 192 \\
\hline $\begin{array}{l}\text { Método de } \\
\text { reconstrução }\end{array}$ & $\begin{array}{l}\text { LOR-RAMLA } \\
\begin{array}{c}(2 \mathrm{i} ; \lambda=0,015 ; \\
\mathrm{a}=2,5 ; \mathrm{I}_{\mathrm{m}}=2 ; \\
\alpha=8,63)\end{array}\end{array}$ & $\begin{array}{c}\text { LOR-RAMLA } \\
(2 \mathrm{i} ; \lambda=0,015 ; \\
\begin{array}{c}\mathrm{a}=2,5 ; \mathrm{I}_{\mathrm{m}}=2 ; \\
\alpha=8,63)\end{array}\end{array}$ & $\begin{array}{l}\text { 2D-OSEM } \\
\text { (8s4i5mm) }\end{array}$ & $\begin{array}{c}\text { Vue Point HD } \\
\text { (3D-ML-OSEM } \\
\text { 32s2i6,4mm) }\end{array}$ & $\begin{array}{c}\text { 3D-OSEM } \\
(8 \mathrm{~s} 4 \mathrm{i} 5 \mathrm{~mm})+ \\
\mathrm{PSF}^{[2]}\end{array}$ & $\begin{array}{c}\text { Vue Point FX } \\
\text { (3D-ML-OSEM } \\
\text { 24s3i5mm)+PSF+ } \\
\text { ToF }{ }^{[3]}\end{array}$ \\
\hline & \multicolumn{6}{|c|}{ TOMOGRAFIA COMPUTADORIZADA DE BAIXA DOSE (Body Low Dose) } \\
\hline $\mathrm{kV}$ & 120 & 120 & 120 & 140 & 130 & 120 \\
\hline $\mathrm{mA}$ & 244 & 80 & $\begin{array}{c}\text { CARE } \\
\text { Dose4D }\end{array}$ & AutomA ${ }^{[4]}$ & CARE Dose4D ${ }^{[4]}$ & AutomA ${ }^{[4]}$ \\
\hline $\begin{array}{l}\text { Espessura do } \\
\text { corte (mm) }\end{array}$ & 5 & 5 & 5 & 3,75 & 5 & 3,75 \\
\hline Matriz & 512 & 512 & 512 & 512 & 512 & 512 \\
\hline
\end{tabular}

Onde: $\mathrm{i}$ = iterações; $\lambda$ = parâmetro de relaxamento; $\mathrm{a}=$ raio da bolha; $\mathrm{I}_{\mathrm{m}}=$ ordem da função Bessel da bolha; $\alpha=$ parâmetro que determina o formato da bolha; $s=$ subconjuntos; $k V=$ tensão elétrica (quilovolts); $\mathrm{mA}=$ corrente elétrica (miliampère).

[1] Todas as instituições habilitam as correções de tempo morto, coincidências aleatórias, atenuação, normalização e decaimento radioativo durante a reconstrução dos dados de PET.

[2] PSF: método de reconstrução que incorpora a função de resposta a um ponto para melhoria da resolução do equipamento ${ }^{10}$.

[3] ToF: tecnologia que permite a mensuração da diferença do tempo de detecção dos dois fótons de aniquilação ${ }^{10}$.

[4] Ferramentas que modificam automaticamente a corrente, de acordo com o tamanho e formato do paciente, visando à redução da dose da tomografia computadorizada.

Figura 4. Parâmetros de aquisição e reconstrução de estudos de PET/CT oncológico utilizados nos SMN investigados. 
A região de interesse ( $\mathrm{ROI}$, Region Of Interest) para análise do SUV é delimitada manualmente (método 2D-manual) por todos os SMN investigados neste estudo. Metade desses emprega, também, o método 3D-semi-automático para delimitação de volumes de interesse (VOI, Volume Of Interest).

Valores de SUV são fortemente dependentes do tamanho e tipo de ROI/VOI. A quantificação do SUV pode variar $0-17 \%$ (para SUV médio) de acordo com o método de delimitação utilizado no processamento ${ }^{5}$. Em um âmbito multicêntrico, é necessário que a metodologia de análise seja, também, realizada de maneira padronizada ${ }^{28}$ para atribuir maior repetibilidade e reprodutibilidade à quantificação do SUV. Dentre os SMN investigados, recomenda-se que, na análise de estudos longitudinais, seja utilizado o método 2D-manual para a delimitação da ROI para o cálculo do SUV, visto que tal método encontra-se disponível nas estações de trabalho de todas as instituições incluídas neste estudo.

Todos os SMN investigados normalizam o SUV pela massa do paciente (SUVBW) e o determinam utilizando o método do valor máximo ( $\left.S U V_{\text {máx }}\right)$. Este método, apesar de refletir o maior valor de atividade metabólica, sofre de uma superestimação devido à natureza de pixel único desta metodologia, o que o torna mais vulnerável ao ruído estatístico inerente à imagem de $\mathrm{PET} / \mathrm{CT}^{29}$. Existem proposições de uso de diferentes métodos de delimitação da ROI/VOI para a determinação do SUV, entre os mais comuns os SUV médio e $S U V_{\text {pico, }}$ métodos menos sensíveis ao ruído das imagens uma vez que incorporam informações de múltiplos pixels/voxels à quantificação ${ }^{5,29}$. Entretanto, estes métodos não estão implementados de forma padronizada na prática clínica ${ }^{5}$, não estando, inclusive, presentes em todas as workstations dos SMN investigados (apenas quatro SMN dispõem destes métodos).

A quantificação do $S U V_{\text {máx }}^{B W}$ pode variar, inclusive, de acordo com a workstation utilizada para a análise. Pierce e colaboradores $(2015)^{30}$ avaliaram a magnitude da variabilidade do SUV decorrente, exclusivamente, da estação de trabalho, utilizando imagens de PET e CT, em formato DICOM, de referência - denominadas Objeto Virtual de Referência (DRO, Digital Reference Object). Foram encontradas variações substanciais entre as workstations analisadas, de magnitude de $-37,8 \%$ a $0 \%$.

Desta maneira, recomenda-se que as instituições utilizem a mesma estação de trabalho para análise de estudos de PET/CT longitudinais (adquiridos unicamente no equipamento da instituição em questão) para que a repetibilidade da quantificação do SUV seja assegurada.

\section{Conclusões}

Todos os SMN implementam programas de garantia da qualidade da instrumentação coerentes com as recomendações nacionais e internacionais.

Observou-se, a partir do levantamento realizado, que os protocolos de aquisição de estudos de PET/CT oncológico dos serviços de PET/CT do estado do Rio Grande do Sul divergem quanto aos parâmetros de aquisição e reconstrução dos dados adquiridos da PET. O grupo de equipamentos analisado contempla três diferentes fabricantes de equipamentos de PET/CT que implementam, por sua vez, algoritmos de reconstrução proprietários ou com parâmetros próprios. Não é possível a definição de um protocolo de aquisição e reconstrução de imagens único para todos os SMN investigados. Desta forma, sugere-se a implementação de uma estratégia de harmonização da quantificação do SUV, de maneira que os diferentes sistemas de PET/CT envolvidos gerem imagens com características equiparáveis.

Há um consenso nos SMN investigados quanto à determinação do SUV pelo método do valor máximo $\left(S U V_{\text {máx }}\right)$, normalizado pela massa do paciente. Visto a vulnerabilidade do SUV aos métodos de delimitação da região de interesse e até mesmo à estação de trabalho utilizada, sugere-se que a análise quantitativa de estudos longitudinais seja realizada: na mesma workstation, caso os exames tenham sido adquiridos no mesmo SMN; utilizando o método 2D-manual para a determinação da região de interesse para o cálculo do SUV, quando os exames tiverem sido adquiridos em diferentes instituições, pois esse é o único método disponível em todos os SMN incluídos neste estudo.

Caso tais recomendações sejam obedecidas, é possível minimizar a influência dos fatores técnicos sobre a quantificação do SUV, atribuindo maior reprodutibilidade e repetibilidade a esta quantificação. Entretanto, além da harmonização da quantificação e da padronização dos métodos de análise das imagens, é de extrema importância que a variabilidade atribuída ao SUV decorrente de fatores biológicos seja minimizada por meio da padronização dos métodos de preparo do paciente.

Como perspectiva futura, sugere-se que este trabalho seja estendido para a inclusão dos fatores biológicos na análise da variabilidade metodológica dentre os SMN, de modo a contemplar todas as etapas do exame de PET/CT oncológico.

\section{Referências}

1. Reinking MF, Osman MM. Prospective Evaluation of Physiologic Uptake Detected with True Whole-Body 18FFDG PET/CT in Healthy Subjects. J Nucl Med Technol2009; 37: 31-37.

2. Jaskowiak CJ, Bianco JA, Perlman SB, Fine JP. Influence of Reconstruction Iterations on 18F-FDG PET/CT Standardized Uptake Values. J Nucl Med. 2005; 46: 424-428.

3. Delbeke D, Coleman RE, Guiberteau MJ, Brow ML, Royal $\mathrm{HD}$, Siegel BA, et al. Procedure Guideline for Tumor Imaging with ${ }^{18}$ F-FDG PET/CT 1.0. J Nucl Med. Mai 2006; 47 (5): 885895.

4. Tan LT, Ong KL. Semi-quantitative Measurements of Normal Organs with Variable Metabolic Activity on FDG PET imaging. Ann Acad Med Singapore. 2011; 33: 183-185.

5. Adams MC, Turkington TG, Wilson JM, Terence ZW. A Systematic Review of the Factors Affecting Accuracy of SUV Measurements. AJR Am J Roentgenol. Ago. 2010; 195 (2): 310-320.

6. Thie JA, Shore B. Understanding the Standardized Uptake Value, Its Methods, and Implications for Usage. J Nucl Med. Set. 2004; 45 (9): 1431-1434.

7. Kinahan PE, Fletcher JW. PET/CT Standardized Uptake Values (SUVs) in Clinical Practice and Assessing Response 
to Therapy. Semin Ultrasound CT MR. Dez 2010; 6 (31): 496505.

8. Geworski L, Knoop BO, Ivancevic V, Bares R, Munz DL. Multicenter comparison of calibration and cross calibration of PET scanners. J Nucl Med. 2002; 43 (5): 635-639.

9. Boellaard R. Standards for PET Image Acquisition and Quantitative Data Analysis. J Nucl Med. 2009; 50: 11S-20S.

10. Boellaard R. Need for Standardization of 18F-FDG PET/CT for Treatment Response Assessments. J Nucl Med. 2011; 52: 93S-100S

11. Makris NE, Huisman MC, Kinahan PE, Lammerstsma AA, Boellaard R. Evaluation of strategies towards harmonization of FDG PET/CT studies in multicentre trials: comparison of scanner validation phantoms and data analysis procedures. Eur J Nucl Med Mol Imaging. Out 2013; 40 (10):1507-15.

12. Boellaard R, Delgado-Bolton R; Oyen WJG, Giammarile F, Tatsch K, Eschner W, et al. FDG PET/CT: EANM procedure guidelines for tumour imaging: version 2.0. Eur J Nucl Med Mol Imaging. 2015; 42: 328-354.

13. ACR-SPR Practice Parameter for Performing FDG-PET/CT in Oncology. Res. 24 - 2012, Amended 2014 (Res. 39). Disponível em: http://www.acr.org/Quality-Safety/StandardsGuidelines/Practice-Guidelines-by-Modality/NuclearMedicine.

14. Amorim BJ. Guideline para PET/CT FDG-18F. Sociedade Brasileira de Medicina Nuclear. Guideline. Abr 2016.

15. NCl Centers of Quantitative Imaging Excellence. CQIE Manual of Procedures; 3.2: Mar 2013.

16. Fukukita H, Suzuki K, Matsumoto K, Terauchi T, Daisaki $H$, Ikari $Y$, et al. Japanese guideline for the oncology FDGPET/CT data. Ann Nucl Med. 2010; 24: 325-334.

17. QIBA Quantitative Imaging Biomarkers Alliance. FDG$\mathrm{PET} / \mathrm{CT}$ as an Imaging Biomarker Measuring Response to Cancer Therapy. Mai 2013; 1.04.

18. IAC - International Accreditation Commission. The IAC Standards and Guidelines for Nuclear/PET Accreditation. Ago 2012.

19. AAPM - American Association of Physicists in Medicine. AAPM Committee Tree: Task Group No. 145 Quantitative Imaging Initiative: Quantitative PET/CT Imaging [Acesso em: Jul 2014]. Colleege Park. 2014. Disponível em: https://www.aapm.org/org/structure/?committee_code=TG1 45.

\section{Contato:}

Andréia Caroline Fischer da Silveira Fischer Serviço de Física Médica e Radioproteção Hospital de Clínicas de Porto Alegre

R. Ramiro Barcelos, 2350 - Bairro Santa Cecilia

Porto Alegre - RS, CEP 90035-903

E-mail: acfischer@hcpa.edu.br
20. Fischer ACFS. Estudo da estratégia de harmonização da quantificação do SUV em imagens de PET/CT. [Dissertação de Mestrado]. Porto Alegre: PUCRS - Faculdade de Engenharia, 2014.

21. Secretaria de Saúde (RS). Anexo II - Resolução no 060/15. Organização da Rede e Regulação do Acesso ao Exame de Tomografia por Emissão de Pósitrons (PET-CT). Diário Oficial do Estado do Rio Grande do Sul. 2015 Abr 16.

22. CNEN - Comissão Nacional de Energia Nuclear. Requisitos de segurança e proteção radiológica para serviços de medicina nuclear. Resolução CNEN 159/13. Dez 13.

23. NEMA - NATIONAL ELECTRICAL MANUFACTURERS ASSOCIATION. NEMA Standards Publication NU 2-2007: Performance Measurements of Positron Emission Tomographs. Rosslyn: NEMA. 2007.

24. Lockhart CM, MacDonald LR, Alessio AM, McDougald WA, Doot RK, Kinahan PE. Quantifying and Reducing the Effect of Calibration Error on Variability of PET/CT Standardized Uptake Value Measurements. J Nucl Med. Fev 2011. 52 (2): 218-224.

25. ANVISA - Agência Nacional de Vigilância Sanitária. Regulamento técnico para instalação e funcionamento de serviços de Medicina Nuclear "in vivo". Resolução $n^{\circ} 38 / 2008$. Jun 08.

26. Lassmann M, Treves ST. Paediatric radiopharmaceutical administration: harmonization of the 2007 EANM paediatric dosage card (version 1.5.2008) and the 2010 North American consensus guidelines. Eur J Nucl Med Mol Imaging. Epub 2014 Mar 06.

27. EARL.eanm.org [página da Internet]. European Association of Nuclear Medicine [acesso em 2016 Abr 19]. Disponível em:

http://earl.eanm.org/cms/website.php?id=/en/projects/fdg_p et ct accreditation.htm.

28. Boellaard R, Krak NC, Hoekstra OS, Lammertsma AA. Effects of Noise, Image Resolution, and ROI Definition on the Accuracy of Standard Uptake Values: A Simulation Study. J Nucl Med. Set 2014; 45 (9): 1519-1527.

29. Lodge MA; Chaudhry MA, Whal RL. Noise considerations for PET Quantification Using Maximum and Peak Standardized Uptake Value. J Nucl Med. Jul 2012; 53: 1041-1047.

30. Pierce II LA, Elston BF, Clunie DA, Nelson D, Kinahan PE. A Digital Reference Object to Analyze Calculation Accuracy of PET Standardized Uptake Value. 